Georgia State University

ScholarWorks @ Georgia State University

\title{
Neural underpinnings of working memory in adult survivors of childhood brain tumors
}

Tricia Z. King

Georgia State University, tzking@gsu.edu

Sabrina $\mathrm{Na}$

Georgia State University, s.diana.na@gmail.com

Hui Mao

Emory University, hmao@emory.edu

Follow this and additional works at: https://scholarworks.gsu.edu/psych_facpub

Part of the Neuroscience and Neurobiology Commons, and the Psychology Commons

\section{Recommended Citation}

King, Tricia Z.; Na, Sabrina; and Mao, Hui, "Neural underpinnings of working memory in adult survivors of childhood brain tumors" (2015). Psychology Faculty Publications. 153.

https://scholarworks.gsu.edu/psych_facpub/153

This Article is brought to you for free and open access by the Department of Psychology at ScholarWorks @ Georgia State University. It has been accepted for inclusion in Psychology Faculty Publications by an authorized administrator of ScholarWorks @ Georgia State University. For more information, please contact scholarworks@gsu.edu. 
Neural Underpinnings of Working Memory in Adult Survivors of Childhood Brain Tumors

\author{
King ${ }^{1}$, Tricia Z., $\mathrm{Na}^{1}$, Sabrina, \& $\mathrm{Mao}^{2}$, Hui \\ ${ }^{1}$ Department of Psychology and Neuroscience Institute, Georgia State University \\ ${ }^{2}$ Department of Radiology and Imaging Sciences, Emory University
}

\begin{abstract}
Author Note
Correspondence concerning this article should be addressed to: Tricia Z. King, tzking@gsu.edu; Georgia State University Department of Psychology, P.O. Box 5010, Atlanta, GA 30302-5010, USA; 404-413-6279; fax: 404-413-6207
\end{abstract}

Abstract Word Count: 202

Manuscript Text Word Count (not including abstract, references, tables and figure): 5768 
King- Working Memory in Brain Tumor Survivors 2

\begin{abstract}
Objective: Adult survivors of childhood brain tumors are at risk for cognitive performance deficits that require the core cognitive skill of working memory. Our goal was to examine the neural mechanisms underlying working memory performance in survivors. Method: We studied the working memory of adult survivors of pediatric posterior fossa brain tumors using a letter nback paradigm with varying cognitive workload (0-, 1-, 2-, and 3-back) and functional magnetic resonance imaging as well as neuropsychological measures. Results: Survivors of childhood brain tumors evidenced lower working memory performance than demographically-matched healthy controls. Whole-brain analyses revealed significantly greater blood-oxygen level dependent (BOLD) activation in the left superior / middle frontal gyri and left parietal lobe during working memory (2-back versus 0-back contrast) in survivors. Left frontal BOLD response negatively correlated with 2- and 3-back working memory performance, Auditory Consonant Trigrams (ACT), and Digit Span Backwards. In contrast, parietal lobe BOLD response negatively correlated with 0-back (vigilance task) and ACT. Conclusions: The results revealed that adult survivors of childhood posterior fossa brain tumors recruited additional cognitive control resources in the prefrontal lobe during increased working memory demands. This increased prefrontal activation is associated with lower working memory performance and is consistent with the allocation of latent resources theory.
\end{abstract}

Key Words: neuroimaging, executive function, posterior fossa, neoplasm, n-back, pediatric 
Neural Underpinnings of Working Memory in Adult Survivors of Childhood Brain Tumors

Advances in diagnosis and treatment have led to improved clinical outcomes of pediatric brain tumor patients. The 5-year survival rate of these patients has increased from $55 \%$ in the 1970s to over 70\% in more recent cohorts (Armstrong et al., 2009; Gurney et al., 2003; Ostrom et al., 2015). With improved treatment outcomes, research on long-term survivorship of childhood brain tumors has reported adverse health, disrupted quality of life, and impaired cognitive and social outcomes (Gurney et al., 2009; Kirchhoff et al., 2011; Robinson, Fraley, Pearson, Kuttesch, \& Compas, 2013). These findings highlight the importance of investigating neural underpinnings associated with childhood brain tumor and its treatment.

Working memory impairment is considered among the primary neurocognitive deficits that contribute to poor long-term outcomes of brain tumor survivors (Palmer, 2008; Wolfe, Madan-Swain, \& Kana, 2012). The core deficits in attention, processing speed, and working memory are associated with treatment-related global cognitive deficits (Dennis et al., 1992; Reeves et al., 2006; Waber et al., 2006). Working memory deficits appear to mediate general cognitive abilities in cancer survivors (Schatz, Kramer, Ablin, \& Matthay, 2000).

Therefore, it is not surprising that working memory is a common deficit among many neurological conditions, such as schizophrenia (Perlstein, Dixit, Carter, Noll, \& Cohen, 2003), multiple sclerosis (Sweet, Rao, Primeau, Durgerian, \& Cohen, 2006), and traumatic brain injury (Medaglia et al., 2012) because working memory is a core executive function and is an essential component for higher-order cognitive processes in humans (Baddeley, 2012; Smith \& Jonides, 1997, 1998). The distributed neural network of the frontal-parietal system has been reported consistently in the functional magnetic resonance imaging (fMRI) literature. Greater prefrontal 
and parietal blood-oxygen level dependent (BOLD) activations observed in fMRI are associated with increased working memory load (Owen, McMillan, Laird, \& Bullmore, 2005).

However, current understanding of the neural mechanisms underlying working memory impairments in adult survivors of childhood brain tumors is limited, and little fMRI research with childhood brain tumor survivors has been reported. To date, two research groups have reported on the feasibility of the letter n-back paradigm in pediatric survivors of childhood brain tumors (Robinson, Pearson, Cannistraci, Anderson, Kuttesch, Wymer, Smith, Park, et al., 2014; Robinson, Pearson, Cannistraci, Anderson, Kuttesch, Wymer, Smith, \& Compas, 2014; Wolfe et al., 2013). In 9 adolescent survivors of pediatric posterior fossa tumors, Wolfe and colleagues (2013) identified a frontal-parietal activation pattern using a contrast of 0-, 1-, and 2-back combined versus fixation. This study demonstrated a broadly typical activation pattern for survivors during the combined vigilance and working memory load n-back task. A control group, however, was not utilized in this study so it is unclear how this activation may differ from neurotypical adolescents.

In a separate study, Robinson and colleagues (2014) examined 17 child and adolescent brain tumor survivors on average 5.64 years post diagnosis of heterogeneous brain tumors and locations. They compared the survivors to 15 similar-age children. Whole-brain examination of the group by n-back load (0-, 1-, 2-, and 3-back) interaction revealed a significant cluster in the left dorsal anterior cingulate cortex (DACC) (Robinson, Pearson, Cannistraci, Anderson, Kuttesch, Wymer, Smith, \& Compas, 2014). More specifically, the survivors showed significant activation in left DACC on the 1- and 2-back relative to baseline, whereas the comparison group showed significant deactivation on these contrasts. Across groups, n-back total accuracy was negatively correlated with BOLD response during the 1-back (-.40) but not correlated with 
King- Working Memory in Brain Tumor Survivors 5

BOLD response during 0-, 2- or 3-back. Furthermore, the left DACC BOLD response during the 1-back positively correlated with parent report of executive dysfunction (i.e., .36; BRIEF Global Executive Composite) and was negatively associated with the WISC-IV Working Memory Index (i.e., -.37). Combined, these findings suggest the importance of separating the role of vigilance (i.e., 1-back, identifying a letter that immediately repeats itself) from working memory in the contribution to clinical measures of executive dysfunction. With this same sample and fMRI data, the authors (Robinson, Pearson, Cannistraci, Anderson, Kuttesch, Wymer, Smith, Park, et al., 2014) took a non-traditional approach of identifying regions of activation in each group separately on specific contrasts (i.e., 2- versus 0-back, and 3- versus 0-back) and then "forceprescribed" these cluster regions onto the other group. Although not a direct comparison between groups at the whole-brain level, activity levels in these prescribed regions were extracted from both groups and correlated with multiple coping and emotional functioning measures. In survivors, the left prefrontal cortex (during 2-back versus 0-back) correlated with ratings of Anxiety/Depression (-.49), Attention Problems (-.61) and Disengagement Coping (-.61). The authors report that the survivors who tended to use disengagement coping were less likely to recruit the frontal regions. In summary, these three studies have shown the feasibility of using the letter n-back paradigm in fMRI studies of survivors of pediatric brain tumors. Each demonstrated traditional areas of activation with this paradigm and highlighted the importance of sample characteristics, level of task difficulty, and diverse analytic approaches. The authors suggest the possibility of frontal dysfunction in survivors but more work is needed directly comparing survivors to healthy controls and deconstructing the meaning of BOLD activation changes with performance correlations. Furthermore, little is known about the neural correlates of working memory BOLD response in long-term adult survivors of pediatric brain tumors. 
The current fMRI study aimed to identify the neural mechanisms underlying working memory difficulty in adult survivors of childhood posterior fossa brain tumors relative to a healthy and demographically-matched control sample. Whole-brain fMRI analyses relative to healthy controls were employed. We found shared or common regions of brain activation in the frontal-parietal working memory network across groups (i.e., conjunction analyses) and therefore confirmed the underlying network was employed similarly across both groups and similar to the n-back literature. In addition, standardized clinical measures of working memory (Auditory Consonant Trigrams and Digit Span Backwards) were examined for group differences and in relationship to the change in BOLD signal in activated regions during the n-back.

\section{Methods}

\section{Participants}

The study was reviewed and approved by the local institutional review boards. Adults who had been treated for a pediatric posterior fossa brain tumor were identified from a large southeastern hospital system database, a previous longitudinal childhood brain tumor study, and an advertisement published in an annual newsletter circulated by the state Brain Tumor Foundation in which survivors were encouraged to call to learn more about the study. Before being enrolled in the study, written consent was provided by all adult participants. Parental consent and participant assent was obtained from two individuals who were 17 years old.

Participants were excluded if English was not their first language, if they met criteria for pervasive developmental disorders, if they indicated a diagnosis of neurofibromatosis, or if they had experienced any other significant neurological insult (e.g., traumatic brain injury). All participants were over the age of 18 (with the exception of two 17-year-old survivors) and were 
at least 5 years past their most recent diagnosis (with the exception of one survivor who was 4.5 years post diagnosis). Information about the brain tumor and treatments were obtained from a thorough retrospective medical records review. None of the survivors had history of progressions or recurrences of their brain tumor. Twenty-seven posterior fossa brain tumor survivors completed both neuroimaging and neuropsychological testing. Ten participants' data were excluded due to either unacceptable motion artifacts in images ( $>3 \mathrm{~mm}, 8$ survivors) or artifact in the cortical regions (2 survivors). In total, 17 adult survivors of posterior fossa tumors were included in the current study. To address potential selection bias, we evaluated whether there were significant differences in behavioral performance when comparing survivors with acceptable imaging and survivors with artifact; $t$-test analyses indicated that there were no significant differences between the two groups.

Control participants were recruited through the institutional psychological research participant pool at a large urban public university and community fliers. Control participants were screened for a history of as well as current psychopathology with the Structured Clinical Interview for DSM-IV-TR Axis 1 (First, Spitzer, Gibbon, \& Williams, 1997). In addition, individuals with a history of neurological or developmental conditions were excluded to ensure that these individuals represented a healthy comparison group. Therefore, no participants in the comparison group had a history of or were diagnosed with Attention Deficit Hyperactivity Disorder (ADHD). Three survivors were diagnosed with acquired ADHD during childhood but none were taking stimulant medications. Cognitive performance and BOLD response data for these three survivors were not outliers, nor appreciably different from the other survivors.

\section{Procedure}




\section{Behavioral Measures}

Auditory Consonant Trigrams (ACT). The ACT (Brown, 1958; Peterson \& Peterson, 1959) was administered to all participants as part of a larger cognitive battery as an out-ofscanner behavioral measure of working memory. Participants were asked to remember three consonants (e.g., B-D-T) that were read by the examiner. They were then asked to count backwards from a given number to prevent rehearsal of the consonants. After 9, 18, or 36 seconds, the participants were asked to recall the three consonants. Performance was computed into Z-scores based on normative data (Stuss, 1987).

Wechsler Memory Scale Digit Span. The Digit Span subtest from the Wechsler Memory Scale (Wechsler, 1997) also was used as a measure of working memory. In addition to the summary normative scaled score, the maximum number of digits recalled on the forward and backward conditions were recorded and analyzed separately, with age co-varied, to differentiate the constructs of attention span (forward condition) and mental manipulation/working memory span (backward condition) within the task.

Wechsler Abbreviated Scale of Intelligence (WASI). The four subtest version (WASI) was used to estimate IQ (Wechsler, 2011): Vocabulary, Similarities, Block Design and Matrix Reasoning. WASI standard scores were used for descriptive purposes only. This measure was not used as a covariate due to previously described limitations of this approach in developmental populations (Dennis et al., 2009).

\section{fMRI Exam with Letter N-Back Working Memory Task Paradigm}

Functional MRI data were acquired using a 3T Siemens Trio MRI scanner using a 12channel head coil. Cushions and forehead straps were placed around each participant's head to 
minimize head movement.

During the n-back task, the participant was asked to monitor a series of letters and to respond yes or no with either his/her index or middle finger on a button box if an item was presented n-items before (ranging from 1 to 3 letters back). A higher 'n' value (i.e., 2-back and 3-back) represented a higher load due to higher monitoring, updating and manipulating demands. In contrast, an 'n' value of 0 (i.e., the 0 -back task) was conceptualized as a measure of vigilance as each participant responded yes or no if a specific letter appeared. In the current study, the 2back versus 0 -back and 3-back versus 0-back contrasts were used to examine working memory more precisely as the common monitoring and vigilance requirements of the tasks were removed.

The task consisted of a block design paradigm with a total of five runs. Eprime (2.0.8) was used for stimuli presentation and accuracy and response time data acquisition. Each run consisted of five different blocks (crosshair, 0-, 1-, 2- and 3-back blocks) and a fixation period (where a cross was presented on the screen). The blocks were counterbalanced between separate runs to minimize order effects. Each block consisted of fifteen letters, where five pre-specified stimuli were the correct targets and the remaining ten letters were non-targets. Each block was preceded by a screen with written instructions for the task lasting $3000 \mathrm{~ms}$, and each letter stimulus was presented for $500 \mathrm{~ms}$ with an inter-stimulus interval of $2500 \mathrm{~ms}$ between each letter presentation. The baseline crosshair-fixation block consisted of a cross on the screen which appeared for $500 \mathrm{~ms}$ at a time, separated by $2500 \mathrm{~ms}$ of blank screen between each cross presentation. Each run lasted approximately four minutes, yielding a total task time of twenty minutes for all five runs. Working memory was defined as the average percent correct of the 2back and 3-back conditions across all five runs, while the vigilance condition was defined as the average percent correct on the 0 -back task across all five runs. 
Participants were trained on the n-back task before they entered the scanner. Participants received a standardized set of instructions on paper. They were given the opportunity to learn the task in an untimed setting and were corrected when they made a mistake. Participants were then administered abbreviated versions of 0 - through 3-back conditions on a laptop connected to a button box identical to the one that was used in the scanner. The stimuli on the screen of the laptop were also identical to the screen projected in the scanner in order to increase familiarity with the format of the task. These steps were taken to ensure that the participants understood the instructions of the task and were familiar with the format and response procedure. This fiveminute procedure did not provide the participants with extensive practice or training prior to the scanner paradigm.

Task-dependent image series were collected using a gradient-recalled T2*-weighted echo-planar-imaging (EPI) sequence based on BOLD contrast. The primary imaging parameters for the BOLD contrast included: 40 slices, $3 \mathrm{~mm}$ slice thickness and $0 \mathrm{~mm}$ slice gap, repetition time $(\mathrm{TR})=2130 \mathrm{~ms}$, echo time $(\mathrm{TE})=30 \mathrm{~ms}$, flip angle $=90$ degrees, nominal resolution $=3 \mathrm{x}$ $3 \times 3 \mathrm{~mm}^{3}$. For anatomical registration, high-resolution T1-weighted structural images were acquired with a 3D magnetization prepared rapid gradient echo (3D MPRAGE) sequence using the following parameters: 176 sagittal slices, field of view $=256 \mathrm{~mm} \times 256 \mathrm{~mm}, 1 \mathrm{~mm}^{3}$ voxel size, $\mathrm{TR}=2250 \mathrm{~ms}, \mathrm{TE}=3.98 \mathrm{~ms}$, inversion time $\mathrm{TI}=850 \mathrm{~ms}$, flip angle $=9$ degrees.

\section{fMRI Data Analyses}

fMRI data analysis was conducted using FEAT (fMRI Expert Analysis Tool) Version 6.01, which is part of FSL (fMRIB's Software Library, www.fmrib.ox.ac.uk/fsl). Data from all five runs were concatenated and preprocessed in the following sequence: motion correction with 
the MCFLIRT tool of FSL; brain extraction using the BET tool of FSL; slice timing correction; spatial smoothing with Gaussian kernel $(\mathrm{FWHM}=5 \mathrm{~mm})$; high pass temporal filtering. The preprocessed data was then registered to its corresponding high resolution T1 MPRAGE images and subsequently to the standard brain template (MNI152 T1 $2 \mathrm{~mm}$ ) using linear boundary based registration (BBR) (Greve \& Fischl, 2009).

General linear modeling was performed using FILM on individual data. A total of 26 regressors were entered into the GLM setup: 13 base regressors (canonical HRF convoluted 0, 1, 2, 3 back timing blocks, crosshair block, instruction block, ISI block, 6 estimated translation and rotation parameters) and their first derivatives. Higher level statistical analyses for contrasts were carried out using FLAME (fMRIB's Local Analysis of Mixed Effects) stage 1. Z statistic images were thresholded using clusters determined by $\mathrm{Z}>1.96$ and a corrected cluster significance threshold of $p=0.05$. Cluster-extent based thresholding was utilized for multiple comparisons correction. Group membership (i.e. survivor vs. control) was entered as a regressor in the group level GLM.

The 2-back versus 0-back and 3-back versus 0-back contrasts were chosen to represent working memory relative to vigilance to elicit brain activation related to working memory neurophysiology. The 2-back versus 0-back and 3-back versus 0-back contrasts were further masked by the 2-back versus crosshair and 3-back versus crosshair contrasts, respectively, to identify regions of positive signal change (i.e. activation rather than deactivation). Given that differences on the 2-back versus 0-back contrast may be due to changes in activation levels on the 0-back condition (rather than the working memory 2-back condition), we deemed it necessary to examine the 0 -back versus crosshair contrast to determine whether BOLD activations in this contrast existed between the two groups. Specifically, we reasoned that 
differences on the 2-back versus 0-back contrast may be interpreted in three possible ways: 1) higher activations on the 2-back task in the survivor group compared to the control group, with identical activations on the 0 -back vigilance task, 2) lower activations on the 0 -back task in the survivor group compared to the control group, with identical activations on the 2-back working memory task and 3) a combination of higher 2-back activation and lower 0-back activation in the survivor group compared to the control group. Given these three possible interpretations, the 0back versus crosshair contrast was probed to determine whether group differences existed on the vigilance task.

Spherical regions of interest (ROIs) were created with a radius of $3 \mathrm{~mm}$ and centered at the coordinates of the peak value of the Z-score. The percentage of the BOLD signal change was obtained with Featquery in these ROIs and correlated with behavioral performance using bivariate Pearson correlations.

Finally, conjunction analyses were performed to determine group similarities in BOLD response to the 2-back versus 0 -back contrast, which is most commonly reported in the literature. Conjunction analyses are used to find common activation patterns across groups by examining voxels that are statistically significant on the contrast in both groups. Conjunction is based on the minimum statistic and tests the null hypothesis that one or more of the effects are null (the “conjunction null”). The conjunction was conducted with FSL easythresh script (http://www2.warwick.ac.uk/fac/sci/statistics/staff/academicresearch/Nichols/scripts/fsl/easythre sh_conj.sh) and tested at a level of $p=0.05$. For more details on the model used and the concepts, procedures and assumptions underlying the model, refer to Price and Friston (1997).

\section{Statistical Analyses for Behavioral Data}


Independent t-tests were used to evaluate whether survivors and controls differed with respect to their performance on all behavioral measures at a significance level of $p<0.05$. Effect sizes were also calculated (Cohen's $d$ ) to illustrate the magnitude of the standardized difference between survivor's and control's performance means.

\section{Results}

Overall, 17 adult survivors and 17 demographically-matched healthy controls were included in the analyses. Groups were matched for gender, age, handedness, and socioeconomic status (see Table 1 for demographic details). Both groups had 11 females and 6 males. Survivors were between 17 and 35 years old, with an average age of 23.2 years $(S D=5.9)$. Survivors were an average of 15.5 years post diagnosis $(S D=7.6$ years $)$. Nine survivors were diagnosed with a high-grade embryonal tumor, while eight survivors were diagnosed with a low-grade astrocytic tumor. Table 1 provides a complete list of the characteristics of the sample.

(Insert Table 1 here)

\section{Behavioral Performance}

We predicted that adult survivors of childhood brain tumor would exhibit lower performance on the n-back fMRI paradigm and standardized clinical measures. Results of onetailed independent t-tests showed that there were significant differences between the survivor and control groups on measures of 2-back performance, 3-back performance, WASI, the longest delay of the ACT (36 second condition), Digit Span performance, and the number of digits 
recalled in the forward condition of Digit Span. On all of these measures, survivors performed significantly lower than controls. The group means, standard deviations, differences in performance on each measure, and the effect sizes of group difference are listed in Table 2.

(Insert Table 2 here)

As a secondary analysis, t-tests were conducted to identify the group differences that existed on the percent change of BOLD signal during the activation and behavioral performance when bifurcating the survivors into the low grade astrocytoma group $(n=8)$ versus the highgrade embryonal tumors $(n=9)$. The two groups were matched for age, age at diagnosis and time since diagnosis.

Results showed that there were significant differences between the groups of high-grade embryonal tumor survivors and low-grade astrocytoma survivors on the behavioral performance of the 3-back task, $t(15)=2.22, p=0.04, d=1.15$. Significant differences were present in the ACT 9s $(t(15)=5.02, p<0.05, d=2.59), 18 \mathrm{~s}(t(15)=2.42, p=0.03, d=1.25)$ and $36 \mathrm{~s}(t(15)=$ 2.42, $p=0.03, d=1.25$ ) measures. There also were significant differences in the Digit Span scaled score $(t(15)=2.86, p=0.01, d=1.48)$, as well as the raw scores of the Digit Span Forward $(t(15)=2.86, p=0.01, d=1.48)$ and Backward $(t(15)=2.20, \mathrm{p}=0.04, d=1.14)$. In all of these analyses, the high-grade embryonal tumor group performed worse than the low-grade astrocytoma group. Analyses of the treatment-related and health-related factors of the two survivor groups indicated that the high-grade embryonal tumor group evidenced higher rates of radiation treatment $\left(\chi^{2}(1, n=17)=9.92, p<.001, \varphi=0.76\right)$, chemotherapy treatment $\left(\chi^{2}(1, n=\right.$ $17)=13.43, p<0.001, \varphi=0.89)$, and endocrine dysfunction $\left(\chi^{2}(1, n=17)=7.24, p<.001, \varphi=\right.$ 0.65) when compared to the low-grade astrocytoma group. As such, the treatment-related and health-related factors related to survivorship of a high-grade embryonal tumor group (i.e. 
radiation, chemotherapy and endocrine dysfunction) were associated with lower cognitive performance on behavioral working memory tasks.

\section{fMRI Results}

We predicted that the neural network recruited in working memory would be disrupted in survivors. Furthermore, we predicted that the correlations between the activation-induced percent change in BOLD signal in prefrontal regions and working memory performance (in and outside of the scanner) would be negatively related.

Whole-brain analyses revealed significant group differences for the 2-back versus 0-back contrast in the left superior frontal/middle frontal gyri and left parietal cortex, with survivors exhibiting higher activations than controls in the cortical structures shown in Figure 1A. The locations and z-values of the peaks and subpeaks that emerged as significantly different between the two groups for the 2-back versus 0 -back contrast are summarized in Table 3 . No group differences were present when evaluating the 0 -back versus crosshair vigilance contrast condition or the 3-back versus 0-back working memory contrast.

(Insert Table 3 here)

(Insert Figure 1 here)

\section{Correlations of Brain Activation and Cognitive Performance}

Mean percent signal changes in the two peak regions, identified by whole-brain analyses (i.e., left superior/middle frontal gyri and left parietal cortex), were examined for their 
relationships with behavioral performance. We predicted that the correlations between the activation induced percent signal change would be negatively related with working memory (convergent support) and less associated with forward attention span or vigilance measures (discriminant support). Pearson bivariate correlations between the signal change and behavioral measures were evaluated to determine whether activation levels in these ROIs in the working memory condition relative to the vigilance condition were correlated with behavioral performances on various working memory measures. Analyses revealed that higher levels of activations in prefrontal regions were associated with lower behavioral performance on higherload working memory tasks. In addition, higher activity in the left parietal cortex was negatively correlated with 0-back overall accuracy and the longest delay of the ACT task (36s). Bivariate correlations between mean percent signal change in the ROIs and behavioral performance are shown in Table 4. Scatterplots of the relationships between working memory tasks and mean percent signal change in the left superior frontal gyrus and the left parietal cortex are represented in Figure $1 \mathrm{~B}$ and 2.

(Insert Table 4 here)

(Insert Figure 2 here)

Secondary analyses were conducted to determine if certain demographic or treatment variables related to behavioral performance and activations in the peak voxels (i.e., left superior frontal gyrus and left parietal cortex). Bivariate correlations indicated that age at testing of the survivor group was significantly correlated to the level of activation in the left superior frontal gyrus, $r(16)=.487, p=.047$. No other demographic or treatment variables were related to the level of activation in either ROI. In addition, age at diagnosis of the survivor group was significantly correlated with behavioral performance on the ACT $(36 \mathrm{~s}), r(16)=.772, p<0.01$, 
but not the other behavioral measures. In short, no demographic variable correlated with n-back performance. However, survivors who were older at the time of their diagnosis performed better on a behavioral measure of working memory. Similarly, time since diagnosis had a statistically significant negative correlation with behavioral performance on the ACT $(36 \mathrm{~s}), r(16)=-.519, p$ $=0.03$. A longer time since diagnosis was related to worse behavioral performance on the working memory measure.

Finally, we conducted t-tests to determine whether the tumor grade (i.e., low grade astrocytomas vs. high grade embryonal tumors) affects activation in the aforementioned left prefrontal and parietal regions. There was no significant difference between the two groups with respect to the percent signal change in the superior frontal gyrus or parietal cortex.

\section{Conjunction Analysis}

Whole-brain analyses revealed regions commonly activated in both the survivor and control groups were in the right superior parietal lobe, right paracingulate gyrus, right insular cortex, left middle frontal gyrus, right and left thalamus and right lateral cerebellum (see Table 5). The pattern of activation for the 2-back versus 0-back contrast showed significant overlap among the survivor group and the control group across the frontal-parietal working memory network. Common areas of activation among the two groups are presented in Figure 2C.

(Insert Table 5 here)

\section{Discussion}


The current study identified significantly greater levels of percent signal change in the BOLD activations of the left prefrontal lobe and left parietal lobe in adult survivors of childhood posterior fossa brain tumors relative to a healthy demographically-similar control group during a verbal working memory task (i.e., working memory 2 -back versus a vigilance 0 -back task). These increased BOLD signals in survivors during working memory reflect that the survivor group recruited greater frontal and parietal activations compared to controls. The increased prefrontal activations are consistent with our predictions based on previous neurological samples with the n-back working memory task; increased prefrontal cortex activation has been reported reliably to be related to the working memory demands of the 2-back task. Furthermore, differences during the 2-back working memory task are due to higher activations in survivors relative to controls, as there were no BOLD differences between the two groups on the 0-back versus crosshair contrast.

The frontal activation is specifically associated with working memory as operationalized by 2-back and 3-back scanner task performance as well as the clinical measures of working memory. In addition, greater left superior frontal region activation was associated with lower performance on the 2-back working memory task, as well as lower performance on the clinical measures of working memory (ACT-36 second delay and Digit Span Backwards). Further supporting the specificity of the working memory association with this frontal activation, the percent change in BOLD activation was not associated with forward digit span or shorter delays of the ACT. In contrast, the percent change in BOLD activation in the parietal region was negatively associated with ACT-36 second delay and 0-back accuracy. Together, these findings suggest that the cognitive demands of the more challenging working memory tasks are 
specifically associated with these increased regions of hemodynamic response and not overall differences in cognitive ability.

Furthermore, the increased parietal lobe activations may be due to increased effort and cognitive demands of verbal working memory, vigilance demands, or even toward basic visual processing of the letters. For instance, it is possible that the upper and lower case changes in the stimuli (e.g., M, t, R, m) elicited greater basic visual processing and phonological working memory demands in the survivors. We developed the stimuli in this manner to ensure that it was a letter/verbal n-back task and to remove the potential confound of shape that sometimes exists when the case of the letter is the same. However, this stimuli specification may have created greater basic visual processing demands that interacted with the working memory demands of the task. It is also possible that increased parietal lobe activations reflect greater vigilance demands. In line with this possibility, behavioral performance on the 0-back (i.e., vigilance task) was significantly correlated with activity in the parietal region, with worse performance associated with higher BOLD signal level. In addition, the upper and lower case stimuli may have also contributed to the greater left-lateralized frontal and parietal working memory network, given the increased phonological verbal working memory. The left hemisphere findings are consistent with prior letter n-back tasks (Owen et al., 2005; Robinson, Pearson, Cannistraci, Anderson, Kuttesch, Wymer, Smith, \& Compas, 2014). However, some researchers have suggested the importance of the right prefrontal lobe due to the novelty of the task demands (Hillary et al., 2011).

Increased prefrontal cortex activation during working memory tasks, as working memory performance declines, has been reported previously in the literature with various neurological populations. Hillary and colleagues suggest that in the context of brain injury, increased BOLD 
in the prefrontal cortex is not due to a compensatory mechanism or brain reorganization but rather attempts of the neurological group to increase cognitive control in the context of poorer performance (Hillary et al., 2011). Our findings are in line with this allocation of latent resources theory of the prefrontal lobe which is observed when prefrontal BOLD response increases with greater task demands. The authors explain that these common findings are not due to compensatory recruitment to maintain task performance or brain reorganization but instead due to the fleeting and typical recruitment of existing resources that is also sometimes observed in healthy non-neurological samples. In our study, the percent change in the prefrontal region related to 2-back performance as well as the longest delay of the Auditory Consonant Trigram task and Digit Span Backwards. Furthermore, this transient allocation of prefrontal cognitive control resources was greater in the adult survivors of childhood posterior fossa tumors. It also appeared that the increased parietal activation was associated with poor performance on the ACT; however, this region was not associated with 3- nor 2-back performance but instead was significantly associated 0-back performance. Consequently, it is important for future studies with survivors of childhood brain tumor and other neurological samples to explore the relationship of BOLD activation differences in these regions with other cognitive performance measures.

Although the primary purpose of the study was to determine cerebral regions that are specific to adult survivors of pediatric brain tumors with respect to BOLD response, it was also deemed important to examine the regions that were being similarly activated in both groups. This analysis was performed to ensure that the regions of the brain recruited by survivors to support their performance on a working memory task are consistent with the working memory networks espoused in studies of neurologically healthy controls (Owen et al., 2005). Indeed, the current study found that the survivors and controls activated the similar, distributed frontal-parietal 
working memory network during the 2 -back versus 0 -back tasks. Comparable activations in the right superior parietal lobe, right paracingulate gyrus, right insular cortex, left middle frontal gyrus, right and left thalamus and right lateral cerebellum were identified in the conjunction analysis. These findings support previous findings and expand to a novel and important group of adult survivors of childhood posterior fossa brain tumors who were on average 15.5 years from diagnosis.

The current results must be considered within the context of the limitations. Although this study was more homogenous in brain tumor location than previous studies (e.g., posterior fossa), approximately half of the sample was high-grade posterior fossa tumor survivors. Therefore, it is possible that there may be differences in neural underpinnings of working memory in survivors with high-grade and more complex treatment histories (e.g., radiation, endocrine treatment) versus those with low-grade cerebellar tumors with surgery only treatment histories. Preliminary analyses comparing these subsamples showed that the high-grade posterior fossa tumor survivors performed worse than low-grade posterior fossa tumor survivors on a variety of behavioral working memory tasks. However, the level to which they activated the frontal and parietal clusters on the 2-back - 0-back contrast was not significantly different, and both groups had large standard deviations. Therefore, small samples such as those in the current study limit the power to detect differences that may exist with a larger sample size. Future studies may focus on a select group such as medulloblastoma or cerebellar juvenile pilocytic astrocytomas and compare functional BOLD activation of each group during working memory tasks to each other as well as to healthy demographically-matched peers.

In addition, this unique sample of long-term survivors was recruited by mailings, newsletters, and word of mouth and may have biased the representativeness of the sample. It is 
possible that survivors with more severe impairments are more eager to participate in clinical research studies. Conversely, it is equally possible that those who are higher functioning are more able to participate and volunteer for studies. Thus, with this design, it is difficult to know how representative this modest sample of long-term survivors is of other posterior fossa survivors. A larger sample would provide greater power to detect important relationships that we may not have found. Likewise, one may also be concerned about differences in the survivors who participated in the study with good versus poor quality imaging data. We explored this possibility, however, and did not find performance differences between these two groups. Therefore, it is important to consider that the results of this study may reflect the best case scenario of working memory performance and underlying neural correlates in pediatric posterior fossa tumor survivors.

It is important to note that the mean IQ of the control group was in the high average range which could reflect the differences between groups that were found. However, on average the nback and other neuropsychological performance was comparable to the performance reported in other fMRI studies (Hillary et al., 2011; Medaglia et al., 2012; Robinson, Pearson, Cannistraci, Anderson, Kuttesch, Wymer, Smith, \& Compas, 2014). The current control group was carefully selected from a larger control sample that was consistent with the state of Georgia census for ethnicity and also included first-generation college students which makes it more consistent with community samples. Larger standard deviations are notable in the survivor group highlighting the increased within group variability across cognitive constructs.

In summary, the current study found that in the context of similar and well-established frontal-parietal working memory network activations, adult survivors of childhood posterior fossa brain tumor also required additional and significantly greater left frontal and left parietal 
cortex activation in response to increased demands of working memory paradigm compared to controls. There was no evidence of compensatory recruitment or brain reorganization; instead the findings are aligned with the allocation of latent resources theory of the prefrontal lobe. Future studies may consider examining how the neural mechanisms identified may relate to cardiorespiratory fitness (i.e., $\mathrm{VO}_{2} / \mathrm{FFM}$ ) (Wolfe et al., 2013) or attention and internalizing symptoms (Robinson, Pearson, Cannistraci, Anderson, Kuttesch, Wymer, Smith, Park, et al., 2014; Robinson, Pearson, Cannistraci, Anderson, Kuttesch, Wymer, Smith, \& Compas, 2014), as these constructs have been suggested as promising lines of research in pediatric short-term survivors of childhood brain tumors. Furthermore, functional and structural connectivity studies may provide complementary information about how the neural regions identified during working memory work together following treatment for pediatric brain tumor. 


\section{Acknowledgements}

We would like to express our gratitude to the individuals and families who participated in this study and generously contributed their time and effort. Also, we would like to acknowledge the Developmental Neuropsychology Research Team for helping with data acquisition and management, the staff at the Georgia State University / Georgia Institute of Technology Joint Center for Advanced Brain Imaging, and the Brain Tumor Foundation for helping share information about this clinical research opportunity with long-term brain tumor survivors. This research was supported by a Research Scholar Grant from the American Cancer Society to TZK (\#RSGPB-CPPB-114044) and a Georgia State University Brains and Behavior Initiative Graduate Student Fellowship (S.N.). No conflicts of interest exist. 


\section{References}

Armstrong, G. T., Liu, Q., Yasui, Y., Huang, S., Ness, K. K., Leisenring, W., . . Packer, R. J. (2009). Long-term outcomes among adult survivors of childhood central nervous system malignancies in the Childhood Cancer Survivor Study. J Natl Cancer Inst, 101(13), 946958. doi: 10.1093/jnci/djp148

Baddeley, A. (2012). Working memory: theories, models, and controversies. Annu Rev Psychol, 63, 1-29. doi: 10.1146/annurev-psych-120710-100422

Brown, J. (1958). Some tests of the decay theory of immediate memory. Quarterly Journal of Experimental Psychology, 10(1), 12-21.

Dennis, M., Francis, D. J., Cirino, P. T., Schachar, R., Barnes, M. A., \& Fletcher, J. M. (2009). Why IQ is not a covariate in cognitive studies of neurodevelopmental disorders. $J$ Int Neuropsychol Soc, 15(3), 331-343. doi: 10.1017/S1355617709090481

Dennis, M., Spiegler, B. J., Obonsawin, M. C., Maria, B. L., Cowell, C., Hoffman, H. J., . . . Ehrlich, R. M. (1992). Brain tumors in children and adolescents--III. Effects of radiation and hormone status on intelligence and on working, associative and serial-order memory. Neuropsychologia, 30(3), 257-275.

First, M. B., Spitzer, R. L., Gibbon, M., \& Williams, J. B. W. (1997). Structured Clinical Interview for DSM-IV axis 1 disorders (SCID-I)-clinical version. Arlington, VA: American Psychiatric Publishing.

Greve, D. N., \& Fischl, B. (2009). Accurate and robust brain image alignment using boundarybased registration. Neuroimage, 48(1), 63-72. doi: 10.1016/j.neuroimage.2009.06.060

Gurney, J. G., Kadan-Lottick, N. S., Packer, R. J., Neglia, J. P., Sklar, C. A., Punyko, J. A., . . . Robison, L. L. (2003). Endocrine and cardiovascular late effects among adult survivors of 
King- Working Memory in Brain Tumor Survivors 26

childhood brain tumors: Childhood Cancer Survivor Study. Cancer, 97(3), 663-673. doi: 10.1002/cncr.11095

Gurney, J. G., Krull, K. R., Kadan-Lottick, N., Nicholson, H. S., Nathan, P. C., Zebrack, B., . . Ness, K. K. (2009). Social outcomes in the Childhood Cancer Survivor Study cohort. J Clin Oncol, 27(14), 2390-2395. doi: 10.1200/jco.2008.21.1458

Hillary, F. G., Medaglia, J. D., Gates, K., Molenaar, P. C., Slocomb, J., Peechatka, A., \& Good, D. C. (2011). Examining working memory task acquisition in a disrupted neural network. Brain, 134(Pt 5), 1555-1570. doi: 10.1093/brain/awr043

Kirchhoff, A. C., Krull, K. R., Ness, K. K., Armstrong, G. T., Park, E. R., Stovall, M., .. . Leisenring, W. (2011). Physical, mental, and neurocognitive status and employment outcomes in the childhood cancer survivor study cohort. Cancer Epidemiol Biomarkers Prev, 20(9), 1838-1849. doi: 10.1158/1055-9965.epi-11-0239

Medaglia, J. D., Chiou, K. S., Slocomb, J., Fitzpatrick, N. M., Wardecker, B. M., Ramanathan, D., . . Hillary, F. G. (2012). The less BOLD, the wiser: support for the latent resource hypothesis after traumatic brain injury. Hum Brain Mapp, 33(4), 979-993. doi:

\subsection{2/hbm.21264}

Ostrom, Q. T., de Blank, P. M., Kruchko, C., Petersen, C. M., Liao, P., Finlay, J. L., . . . Barnholtz-Sloan, J. S. (2015). Alex's Lemonade Stand Foundation Infant and Childhood Primary Brain and Central Nervous System Tumors Diagnosed in the United States in 2007-2011. Neuro Oncol, 16 Suppl 10, x1-x36. doi: 10.1093/neuonc/nou327

Owen, A. M., McMillan, K. M., Laird, A. R., \& Bullmore, E. (2005). N-back working memory paradigm: a meta-analysis of normative functional neuroimaging studies. Hum Brain Mapp, 25(1), 46-59. doi: 10.1002/hbm.20131 
Palmer, S. L. (2008). Neurodevelopmental impact on children treated for medulloblastoma: a review and proposed conceptual model. Dev Disabil Res Rev, 14(3), 203-210. doi: $10.1002 /$ ddrr.32

Perlstein, W. M., Dixit, N. K., Carter, C. S., Noll, D. C., \& Cohen, J. D. (2003). Prefrontal cortex dysfunction mediates deficits in working memory and prepotent responding in schizophrenia. Biol Psychiatry, 53(1), 25-38.

Peterson, L. R., \& Peterson, M. J. (1959). Short-term retention of individual verbal items. J Exp Psychol, 58, 193-198.

Price, C. J., \& Friston, K. J. (1997). Cognitive conjunction: A new approach to brain activation experiments. Neuroimage, 5, 261-270.

Reeves, C. B., Palmer, S. L., Reddick, W. E., Merchant, T. E., Buchanan, G. M., Gajjar, A., \& Mulhern, R. K. (2006). Attention and memory functioning among pediatric patients with medulloblastoma. J Pediatr Psychol, 31(3), 272-280. doi: 10.1093/jpepsy/jsj019

Robinson, K. E., Fraley, C. E., Pearson, M. M., Kuttesch, J. F., Jr., \& Compas, B. E. (2013). Neurocognitive late effects of pediatric brain tumors of the posterior fossa: a quantitative review. J Int Neuropsychol Soc, 19(1), 44-53. doi: 10.1017/s1355617712000987

Robinson, K. E., Pearson, M. M., Cannistraci, C. J., Anderson, A. W., Kuttesch, J. F., Jr., Wymer, K., . . Compas, B. E. (2014). Functional neuroimaging of working memory in survivors of childhood brain tumors and healthy children: Associations with coping and psychosocial outcomes. Child Neuropsychol, 1-24. doi: 10.1080/09297049.2014.924492

Robinson, K. E., Pearson, M. M., Cannistraci, C. J., Anderson, A. W., Kuttesch, J. F., Wymer, K., . . Compas, B. E. (2014). Neuroimaging of executive function in survivors of 
pediatric brain tumors and healthy controls. Neuropsychology, 28(5), 791-800. doi: $10.1037 /$ neu0000077

Schatz, J., Kramer, J. H., Ablin, A., \& Matthay, K. K. (2000). Processing speed, working memory, and IQ: a developmental model of cognitive deficits following cranial radiation therapy. Neuropsychology, 14(2), 189-200.

Smith, E. E., \& Jonides, J. (1997). Working memory: a view from neuroimaging. Cogn Psychol, 33(1), 5-42. doi: 10.1006/cogp.1997.0658

Smith, E. E., \& Jonides, J. (1998). Neuroimaging analyses of human working memory. Proc Natl Acad Sci U S A, 95(20), 12061-12068.

Stuss, D. T. (1987). Contribution of frontal lobe injury to cognitive impairment after closed head injury: Methods of assessment and recent findings.

Sweet, L. H., Rao, S. M., Primeau, M., Durgerian, S., \& Cohen, R. A. (2006). Functional magnetic resonance imaging response to increased verbal working memory demands among patients with multiple sclerosis. Hum Brain Mapp, 27(1), 28-36. doi: 10.1002/hbm.20163

Waber, D. P., Pomeroy, S. L., Chiverton, A. M., Kieran, M. W., Scott, R. M., Goumnerova, L. C., \& Rivkin, M. J. (2006). Everyday cognitive function after craniopharyngioma in childhood. Pediatr Neurol, 34(1), 13-19. doi: 10.1016/j.pediatrneurol.2005.06.002

Wechsler, D. (1997). Wechsler Memory Scale-Third edition. Administration and scoring manual. San Antonio, TX: The Psychological Corporation.

Wechsler, D. (2011). Wechsler Abbreviated Scale of Intelligence- Second Edition Manual. Bloomington, MN: Pearson. 
Wolfe, K. R., Madan-Swain, A., Hunter, G. R., Reddy, A. T., Banos, J., \& Kana, R. K. (2013). An fMRI investigation of working memory and its relationship with cardiorespiratory fitness in pediatric posterior fossa tumor survivors who received cranial radiation therapy. Pediatr Blood Cancer, 60(4), 669-675. doi: 10.1002/pbc.24331

Wolfe, K. R., Madan-Swain, A., \& Kana, R. K. (2012). Executive dysfunction in pediatric posterior fossa tumor survivors: a systematic literature review of neurocognitive deficits and interventions. Dev Neuropsychol, 37(2), 153-175. doi:

$10.1080 / 87565641.2011 .632462$ 
Table 1

Demographic, Diagnostic and Treatment Variables of the Sample

Demographic Variables

Survivors $(n=17) \quad$ Controls $(n=17)$

\begin{tabular}{lcc}
\hline Female $(n, \%)$ & $11(65 \%)$ & $11(65 \%)$ \\
Hollingshead socio-economic status^ (mean $\pm S D)$ & $2.27 \pm 1.33$ & $2.56 \pm 1.09$ \\
Age at testing (mean $\pm S D)$ & $23.39 \pm 4.46$ & $23.24 \pm 5.89$ \\
Range & $17-35$ & $18-35$ \\
Median (interquartile range) & $21(18.5-28.5)$ & $22(20-25)$ \\
Ethnicity $(n, \%)$ & $16(94 \%)$ & $3(18 \%)$ \\
Caucasian & $1(6 \%)$ & $1(6 \%)$ \\
African-American & $0(0 \%)$ & $16(94 \%)$ \\
Other & & $1(6 \%)$ \\
Employment status $(n, \%)$ & $1(6 \%)$ & $0(0 \%)$ \\
Student & $9(53 \%)$ & $0(0 \%)$ \\
Employed & $6(35 \%)$ & \\
Unemployed & $1(6 \%)$ & \\
Disability & $18 \%)$ &
\end{tabular}

Diagnostic Variables

Years post diagnosis (mean $\pm S D)$

$15.5(7.6)$

Range

$4.5-30$

Median (interquartile range)

$14.94(8.08-21.78)$

Age at diagnosis (mean $\pm S D$ )

$7.65(4.90)$ 
Range

Median (interquartile range)

Tumor type (n, \%)

High-grade embryonal tumors ${ }^{\dagger}$

Low-grade astrocytic tumor ${ }^{\Delta}$

Treatment Variables

Radiation type ( $n, \%)$

No radiation

Focal

Whole-brain

Craniospinal with boost

Chemotherapy $(n, \%)$

Neurosurgery

Hydrocephalus (n, \%)

Seizure Medication ( $n, \%)$

Endocrine Dysfunction ( $n, \%)$
$1-17$

$7(3.5-10.5)$

$9(53 \%)$

$8(47 \%)$

$8(47 \%)$

$1(6 \%)$

$0(0 \%)$

$8(47 \%)$

$8(47 \%)$

$17(100 \%)$

$11(65 \%)$

$0(0 \%)$

$8(47 \%)$

Note. ${ }^{\wedge} \mathrm{SES}=$ Current socioeconomic status, calculated using the Hollingshead Four Factor Index of Social Status (Hollingshead, 1975). Family SES was used in instances where the individual reported being financially dependent on their family.

${ }^{\dagger} 8$ Medulloblastoma, 1 PNET, NOS; ${ }^{\Delta} 7$ Juvenile pilocytic astrocytoma, 1 Fibrillary astrocytoma 
Table 2

Performance Measures of the Survivor and Control Groups

\begin{tabular}{|c|c|c|c|c|}
\hline & $\begin{array}{l}\text { Survivor group } \\
\qquad n=17\end{array}$ & $\begin{array}{l}\text { Control group } \\
\qquad \begin{array}{l}n=17\end{array}\end{array}$ & & \\
\hline & Mean $(S D)$ & Mean $(S D)$ & $t$ & Cohen's $d$ \\
\hline 0-back Accuracy (\%) & $96.40(3.52)$ & $97.73(2.14)$ & 1.33 & -0.46 \\
\hline 1-back Accuracy (\%) & $93.80(7.13)$ & $96.55(3.83)$ & 1.40 & -0.48 \\
\hline 2-back Accuracy (\%) & $86.59(7.05)$ & $92.39(5.12)$ & $2.75 * *$ & -0.94 \\
\hline 3-back Accuracy (\%) & $83.45(6.62)$ & $91.53(6.04)$ & $3.72 * *$ & -1.28 \\
\hline WASI standard score & $100.12(11.75)$ & $112.00(7.33)$ & $3.54 * *$ & -1.21 \\
\hline AC T 9s (z-score) & $0.09(0.95)$ & $0.38(1.12)$ & 0.82 & -0.33 \\
\hline ACT 18s (z-score) & $-0.25(1.13)$ & $0.23(0.81)$ & 1.46 & -0.49 \\
\hline ACT 36s (z-score) & $-0.07(1.14)$ & $0.96(0.86)$ & $2.99 * *$ & -1.02 \\
\hline Digit Span (scaled score) & $8.47(2.50)$ & $11.29(1.99)$ & $3.64 * *$ & -1.25 \\
\hline Forward Digits (raw) ${ }^{\wedge}$ & $5.94(1.34)$ & $7.29(1.10)$ & $3.21 * *$ & -1.10 \\
\hline Backward Digits (raw) & $4.53(1.23)$ & $5.35(1.17)$ & 2.00 & -0.68 \\
\hline Superior frontal gyrus activation & $0.39(0.30)$ & $0.04(0.19)$ & $-4.12 * *$ & 1.39 \\
\hline Left parietal lobe activation & $0.68(0.50)$ & $0.13(0.27)$ & $-4.00 * *$ & 1.37 \\
\hline
\end{tabular}


Note. * indicates significance of $\mathrm{t}($ one-tailed), $p<.05, * *$ indicates significance of $p<.01$; with regard to directionality, we hypothesized that survivors would perform lower than controls on all measures; ${ }^{\wedge}$ Forward digits performance remained significantly different between both groups after adding age as a covariate. Activation = percent change in BOLD signal on the 2-back condition as compared to the 0 -back condition. $\mathrm{ACT}=$ Auditory Consonant Trigrams 
Table 3

Location of Significant Working Memory Activation Differences between Survivors and Controls: Peaks and Subpeaks for the 2-Back versus 0-Back Contrast

MNI Coordinates

Anatomical Region

\begin{tabular}{lllllll} 
Cluster & Cluster $P$ & Peak & & & & \\
\cline { 4 - 6 } & & & & & & \\
Size & (corrected) & $Z$-value & $x$ & $y$ & $z$
\end{tabular}

(voxels)

Left Superior Frontal Gyrus

Left Middle Frontal Gyrus

Left Superior Frontal Gyrus

Left Superior Frontal Gyrus

Left Middle Frontal Gyrus

Left Parietal Lobe/Precuneus

Left Parietal Lobe/Precuneus

Left Parietal Lobe/Precuneus

Left Parietal Lobe/Inferior Parietal Lobule

Left Parietal Lobe/Inferior Parietal Lobule
1088

0.00702

3.59

3.48

$\begin{array}{llll}3.46 & -30 & 30 & 52\end{array}$

$\begin{array}{llll}3.43 & -6 & 18 & 54\end{array}$

$\begin{array}{llll}2.97 & -26 & 22 & 54\end{array}$

840

0.032

3.78

$-30$

$-74 \quad 46$

$\begin{array}{llll}3.78 & -32 & -70 & 44\end{array}$

$\begin{array}{llll}3.21 & -38 & -78 & 40\end{array}$

$\begin{array}{llll}2.96 & -46 & -62 & 48\end{array}$

$\begin{array}{llll}2.77 & -42 & -66 & 50\end{array}$

Peak voxel coordinated (MNI space) and statistical values $(Z>1.96)$ are listed for significant clusters $\left(P_{\text {cluster size corrected }}<0.05\right)$. 
Table 4

Bivariate Correlations between Mean Percent Signal Change and Behavioral Performance

1. 2.

1. Superior frontal gyrus activation

2. Parietal lobe activation

0.13

0-back percent correct

$-0.13$

$-0.35^{*}$

1-back percent correct

$-0.20$

0.05

2-back percent correct

$-0.42 *$

$-0.18$

3-back percent correct

$-0.48 * *$

$-0.29$

ACT 9s Z-score

$-0.10$

$-0.16$

ACT 18s Z-score

$-0.18$

ACT 36s Z-score

$-0.34 *$

$-0.42 *$

Digit span Scaled Score

$-0.34 *$

Digit Span Forward Digits^

$-0.27$

$-0.34$

Digit Span Backward Digits^

$-0.36^{*}$

$-0.29$

Note. $*$ indicates significance at the 0.05 level, ** indicates significance at the 0.01 level;

$\wedge r$ values are partial correlations after covarying for age

Table 5 
King- Working Memory in Brain Tumor Survivors 36

Location of Significant Working Memory Regions of Activation Conjunction among Survivors and Controls for the 2-Back versus 0-Back Contrast

Cluster

MNI Coordinates

Anatomical Region

Size

Cluster $P$

Peak

(voxels)

Z-value

$x$

$y \quad z$

Right Superior Parietal Lobe

6335

$<0.001$

5.31

$44 \quad-46$

56

Right Paracingulate Gyrus

5790

$<0.001$

5.31

$\begin{array}{lll}4 & 24 & 48\end{array}$

Left Middle Frontal Gyrus

3562

$<0.001$

4.73

$-26$

$10 \quad 50$

Right Insular Cortex

508

0.02

4.83

$\begin{array}{lll}32 & 22 & 0\end{array}$

Right Thalamus

506

0.02

3.69

$\begin{array}{lll}8 & -6 & 6\end{array}$

Right Cerebellum

471

0.03

4.59

34

$-64$

$-26$

Left Thalamus

462

0.03

3.62

$\begin{array}{lll}-8 & -12 & 8\end{array}$

Peak voxel coordinated (MNI space) and statistical values $(Z>1.96)$ are listed for significant clusters $\left(P_{\text {cluster size corrected }}<0.05\right)$. 


\section{Figure Legends}

Figure 1. (A) Areas of significant working memory BOLD activation differences between survivors and controls on the 2-back versus 0-back contrast. Survivors exhibited higher activations than healthy controls in left superior frontal gyrus and parietal cortex regions. See Table 3 for cluster size and peak MNI coordinates. B) Scatterplots are displayed to illustrate relationships between mean percent signal change in frontal and parietal ROIs and n-back performance for survivors and controls. Left parietal cortex activation was negatively correlated with 0 -back performance only $r(32)=-0.35, p<0.05$. Left superior frontal gyrus activation was negatively correlated with the 2-back $(r(32)=-0.42, p<0.05)$ and 3-back conditions $(r(32)=-$ $0.48, p<0.01$ ) but not the lower load n-back conditions. C) Significant regions of similar working memory BOLD activation among survivors and controls on the 2-back versus 0-back contrast. Conjunction analyses indicated similar bilateral activations in frontal and parietal lobes, thalamus and cerebellum. See Table 5 for cluster size and peak MNI coordinates. All brain images are presented in radiological space. Activation maps were superimposed on a T1weighted standard MNI152 brain template. Color bar indicates Z-values (results thresholded at $p$ $=0.05$, cluster-based corrections).

Figure 2. Scatterplots of mean percent signal change in the left superior frontal gyrus ROI and behavioral performance on working memory tasks for survivors and healthy controls. A) Left superior frontal gyrus activation was negatively correlated with the longest delay condition of the Auditory Consonant Trigram task $r(32)=-0.34, p<0.05$. This ROI was not significantly correlated with the $9 \mathrm{~s}(r(32)=-0.10, p>0.05)$ and $18 \mathrm{~s}(r(32)=-0.18, p>0.05)$ shorter delay 
conditions of the ACT task. B) Left superior frontal gyrus activation was negatively correlated with the Digit Span Backward Digits measure, $r(32)=-0.36, p<0.05$, but not the Forward Digits measure, $r(32)=-0.27, p<0.05$. These findings were consistent after covarying for age. 
Figure 1.
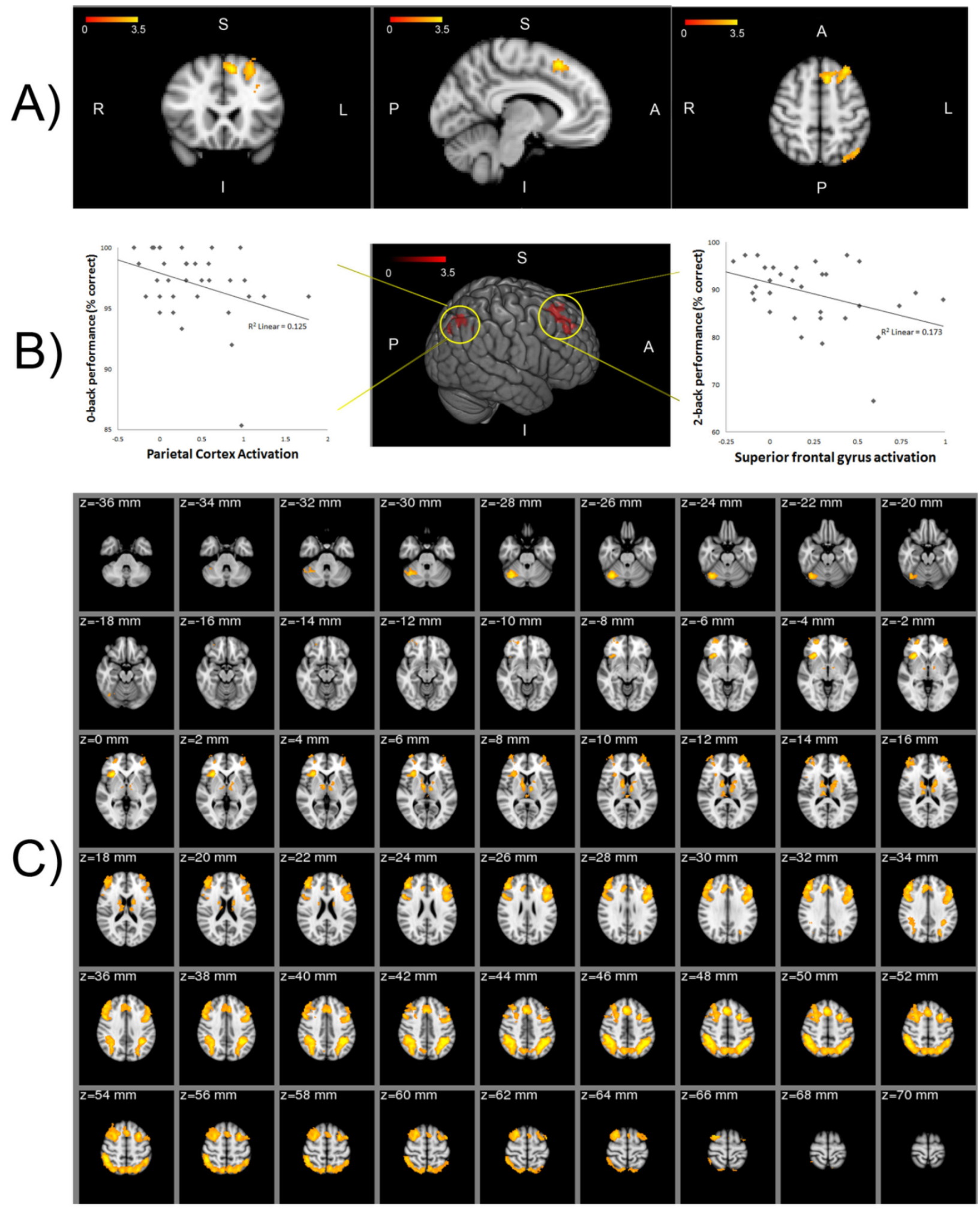
Figure 2.

A)
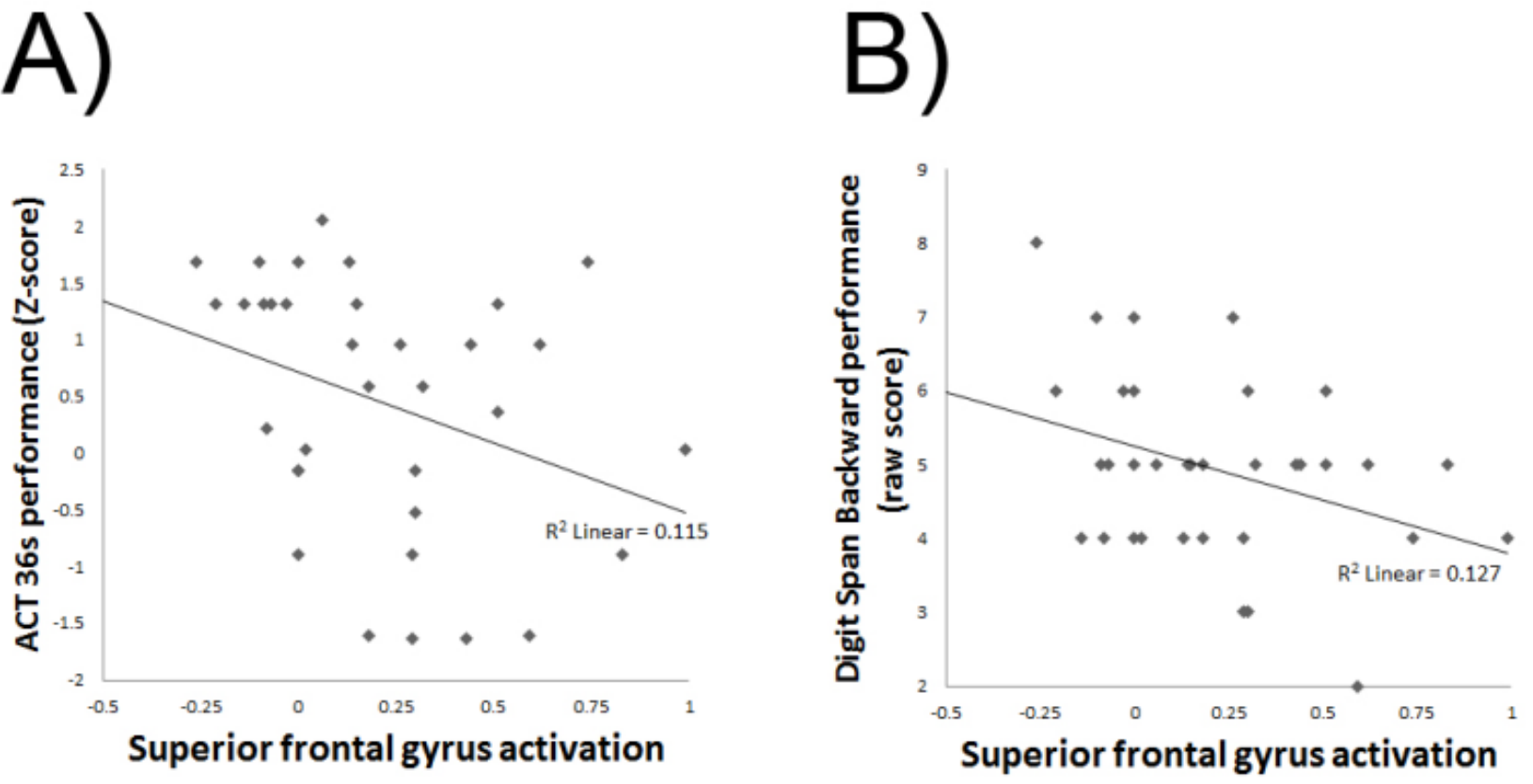